\title{
EL RENDIMIENTO ACADÉMICO DE LOS ESTUDIANTES EN SITUACIÓN DE DISCAPACIDAD
}

THE ACADEMIC PERFORMANCE OF STUDENTS IN A SITUATION OF

\author{
DISABILITY
}

Mario G. Oloriz ${ }^{1}$, Juan Manuel Fernández ${ }^{2}$

Enviado: 29 de junio de 2020

Aceptado: 8 de junio de 2020

\footnotetext{
${ }^{1}$ Magister en Gestión Universitaria, Departamento de Ciencias Básicas, Universidad Nacional de Luján, moloriz@unlu.edu.ar

2 Secretario Académico, Departamento de Ciencias Básicas, Universidad Nacional de Luján, jmfernandez@unlu.edu.ar
} 


\section{Resumen}

El rendimiento académico de los estudiantes, principalmente durante los primeros años de estudios universitarios, es uno de los factores con mayor incidencia respecto del abandono educativo. Se caracterizó el abandono en la Universidad Nacional de Luján (UNLu), analizando las posibles causas del fenómeno y estudiando el impacto de los Programas de Becas respecto de la mejora en la retención y el rendimiento académico de los estudiantes. La UNLu ha implementado desde el año 2008 una Comisión Permanente de Seguimiento de la Accesibilidad que tiene por finalidad contribuir a superar las barreras a la accesibilidad y la promoción de los estudiantes con discapacidad. Se abordó el estudio del rendimiento académico y abandono de aquellos ingresantes que declaran alguna situación de discapacidad al momento de su inscripción. Se trabajó con los ingresantes de las cohortes 2010 a 2015, encontrando un mejor desempeño de los estudiantes que se declararon en situación de discapacidad respecto de los demás integrantes de la misma corte, tanto en su desempeño académico como en la tasa de abandono y la tasa de graduación.

Palabras clave: Situación

Discapacidad, Becas, Accesibilidad, Abandono.

\section{Abstract \\ The academic performance of} the students, mainly during the first years of university studies, is one of the factors with the highest incidence regarding educational dropout. Abandonment at the National University of Luján (UNLu), was characterized analyzing the possible causes of the phenomenon and studying the impact of the Scholarship Programs regarding the improvement in student retention and academic performance. The UNLu has implemented since 2008 a Permanent Accessibility Monitoring Commission that aims to help overcome barriers to accessibility and the promotion of students with disabilities. The study of academic performance and abandonment was addressed of those entrants who 
declare a disability situation at the time of enrollment.

We worked with the entrants of the cohorts 2010 to 2015, finding a better performance of the students who declared themselves disabled compared to the other members of the same court, both in their academic performance and in the

\section{INTRODUCCIÓN}

El desarrollo y aplicación de políticas que mejoren el desempeño de los estudiantes que se encuentran con barreras tanto físicas como psíquicas para el cursado de estudios universitarios son medidas que más allá de mejorar la retención de estos estudiantes ayudan a equilibrar las diferencias que existen en término de las oportunidades con que cuentan los miembros de la sociedad provenientes de sectores más desfavorecidos.

Mediante el estudio cuantitativo de la actividad académica de los estudiantes que integraron las cohortes 2007-2010, se verificó que el Régimen de Becas Estudiantiles de la UNLu es una herramienta eficaz para la disminución del abandono al comprobar una menor tasa en los estudiantes que participan del abandonment rate and the graduation.

Keyword: Disability Situation, Scholarships, Accesibilit, Abandonment.

Programa de Becas para Estudiantes respecto de la observada en aquellos estudiantes que no accedieron a este beneficio. La diferencia entre la tasa de abandono de quienes participan del Programa de Becas y quienes no es de alrededor del $36 \%$, a favor de quienes obtuvieron algún tipo de beca. (Oloriz, Fernandez y Amado, 2013)

El principal impacto del Programa de Becas se da en el abandono temprano, aquel que se produce durante los dos primeros años de estudio, dado que la tasa de abandono temprano de quienes participan del Programa es del orden del 19\% mientras que para los demás estudiantes es superior al $48 \%$.

En el mismo estudio el abandono que se produce entre quienes acceden al Programa de Becas de la UNLu tiene una estrecha relación con el monto que 
perciben como estipendio o ayuda económica los becarios. Dado que existen distintas categorías de beca en este Programa, se observó que a mayor monto que percibe el becario menor es la tasa de abandono. Con lo cual, podría decirse que la probabilidad de abandono es inversamente proporcional al monto que el becario percibe como estipendio. (Oloriz, Fernandez y Amado: 2013: Tabla 5).

Además, los programas de becas de carácter nacional existentes a ese momento (PNBB, PNBU, PNBTIC'S, BBAPRO), con financiamiento externo a la universidad, también resultan eficientes para mejorar la retención estudiantil y disminuir el abandono. (Oloriz, Fernandez y Amado, 2014).

En el año 2014, más precisamente el día 20 de Febrero de 2014, se puso en marcha el Programa de Respaldo a Estudiantes Argentinos (PROG.R.ES.-AR.), cuyo objetivo es la inclusión social de los jóvenes entre 18 y 24 años de edad que continúen estudios superiores, por parte del Gobierno Nacional. (Decreto 84/2014Presidencia de la Nación Argentina).

Durante el año 2015, la evaluación del impacto de dicho programa en la disminución del abandono en la UNLu, demostró que la tasa de abandono de los jóvenes entre 18 y 24 años que ingresaron a esta universidad en el año 2014, fue menor para quienes accedieron al PROG.R.ES.AR., en casi 13 puntos porcentuales, respecto de la media de abandono para esa cohorte. (Oloriz, Fernandez y Rodríguez, 2015).

Luego de haber comprobado la eficiencia del Programa de Becas Estudiantiles de la Universidad Nacional de Luján (UNLu) para reducir el abandono de los estudiantes que provienen de los sectores sociales de menores ingresos, se estudió el resultado de las acciones que viene implementando esta Universidad Nacional para mejorar las condiciones en que cursan los ingresantes que se encuentran en situación de discapacidad.

Según lo publicado en el Sitio Web del Ministerio de Educación y Deportes de la Nación de la República Argentina $^{3}$, durante el año 2012 se realizó el primer relevamiento de estudiantes en situación de discapacidad en las universidades argentinas. Del mismo surgió que, durante ese año, concurrían a las universidades de gestión pública 1.064

\footnotetext{
${ }^{3}$ www.argentina.gov.ar/educación/
} 
estudiantes en situación de discapacidad del total de 1.394.782 declarados por las mismas universidades (Lo que representa el 0,076\% del total). En el 2013, se repite el relevamiento del cual surgen 808 estudiantes más en situación de discapacidad.

Comparativamente, en el período 2004-2005 se realiza un relevamiento en las universidades españolas, en instituciones públicas y privadas, encontrando que concurrían 7.739 estudiantes con discapacidad lo que representaba el $0,52 \%$ de los estudiantes universitarios del mismo período. (Peralta; 2007)

Existen cuatro elementos clave como definitorios en las prácticas de inclusión de los grupos en desventaja, según lo identificado por la UNESCO.

En primer término, se debe reconocer a la inclusión como un proceso mediante el cual se deberán buscar, de manera interminable, mejores formas de respuesta a la diversidad. El segundo elemento, se refiere a que la inclusión supone la identificación y eliminación de barreras $\mathrm{y}$, por lo tanto, es necesario relevar, relacionar y evaluar información para planificar mejoras en las políticas y prácticas institucionales que permitan ir quitando estas barreras. El tercero de los elementos, determina que la inclusión tiene relación con la presencia, participación y éxito escolar de todos los estudiantes. El último elemento señala que la inclusión implica un énfasis especial en los grupos de estudiantes que pueden estar en riesgo de marginación, exclusión o fracaso escolar y supone la responsabilidad moral de asegurar se tomen las medidas necesarias para garantizar la presencia, participación y éxito escolar en el sistema educativo de estos grupos. (UNESCO, 2003)

En este sentido, en la República Argentina, la Ley de Educación Superior postula en el inciso d) de su artículo $2^{\circ}$ : "Establecer las medidas necesarias para equiparar las oportunidades y posibilidades de las personas con discapacidades permanentes o temporarias" (Ley 24.521; 1995). Por otra parte, en el año 2002, se introdujo una modificación a esta Ley por la cual se incorpora como uno de los derechos de los estudiantes de instituciones estatales, o de gestión pública, en el inciso f) del artículo $13^{\circ}$ : "Las personas con discapacidad, durante las evaluaciones, deberán contar con los servicios de interpretación y los apoyos técnicos necesarios y suficientes". Con esta modificación, se busca propender a la 
igualdad de oportunidades y retiro de las barreras a las que hace alusión el documento de la UNESCO.

Posteriormente, en el año 2006, se promulga en Argentina la Ley de Educación Nacional, la cual abarca todos los niveles educativos que conforman el sistema de educación nacional, la cual establece como uno de los fines de las políticas educativas nacionales: "Brindar a las personas con discapacidades, temporales o permanentes, una propuesta pedagógica que les permita el máximo desarrollo de sus posibilidades, la integración y el pleno ejercicio de sus derechos" (Ley 26.206: Inciso n, Art.11, 2006). Esta Ley postula, en varios artículos, que se deben desarrollar estrategias educativas tendientes a la integración y eliminación de las barreras que existen para el acceso y permanencia a todos los niveles educativos a las personas en situación de discapacidad.

En el año 2008 fue ratificada, por Ley 26.378, la vigencia de la Convención de los Derechos de las Personas con Discapacidad (ONU, 2006), adhiriendo al Protocolo Facultativo de la misma, otorgando así fuerza de ley y cumplimiento obligatorio a todas sus disposiciones, por parte del Parlamento Nacional.

\section{Contexto Institucional}

Políticas de apoyo a estudiantes con discapacidad, otorgando becas a estudiantes en esa situación, fueron sostenidas por la UNLu desde el año 1993.

En el año 2008, se crea la Comisión Permanente de Seguimiento de la Accesibilidad, con la misión de llevar adelante cuatro líneas programáticas destinadas a mejorar las condiciones de acceso, permanencia y logro de objetivos de las personas con discapacidad en la Universidad, por parte del Consejo Superior (Res. 660/08). Se crea e integra la Comisión con una amplia representación de los diferentes sectores involucrados (políticos, administrativos y académicos). Dada la complejidad del trabajo a abordar por esta Comisión, se definieron las siguientes líneas programáticas de trabajo: Accesibilidad Física, Accesibilidad Comunicacional, Accesibilidad Pedagógica y Accesibilidad Laboral (UNLu, 2008).

Por otra parte, también integraron la Comisión Interuniversitaria de Discapacidad y Derechos Humanos, que actualmente forma parte de la Red Bien del Consejo Interuniversitario Nacional, y otras redes latinoamericanas, y participaron de acciones y programas del Ministerio de 
Educación de la Nación, miembros de la Comisión Permanente.

Para el cumplimiento de los objetivos asignados se hizo necesario el relevamiento de los estudiantes, docentes y nodocentes que declararan situación de discapacidad. Se optó por el criterio de la declaración voluntaria de la situación de discapacidad, comenzando el relevamiento de los estudiantes en las instancias de ingreso a la Universidad.

Se adoptó la filosofía de la accesibilidad universal y se asumieron los criterios elaborados por la Convención de los Derechos de las Personas con Discapacidad aprobados por la ONU en el año 2006 y ratificados a través del Protocolo Facultativo por el Parlamento Nacional, con carácter de Ley en el año 2008. (ONU, 2006)

Para garantizar la accesibilidad física, se desarrollaron acciones centradas en los aspectos edilicios, asegurando la existencia de un "corredor accesible" que recorre el campus de la Sede Central de la UNLu posibilitando a las personas con diferentes discapacidades el acceso a las principales dependencias (aulas, biblioteca, comedor, dependencias administrativas, auditorio, servicios médicos, etc.), todo coordinado desde la Comisión. Para ello, se realizó un relevamiento integral de los más de 30 edificios que posee la Universidad en todas sus Sedes y Delegaciones, estableciendo un plan de obras a desarrollar a mediano plazo.

Como paso previo, se capacitó a los arquitectos responsables de la elaboración de los planos y contrataciones en los criterios $y$ normativas de accesibilidad aplicables a los diferentes proyectos.

En términos del eje comunicacional, se ha garantizado el cumplimiento de los estándares internacionales de accesibilidad en el sitio web de la Universidad ${ }^{4}$, y se difundieron programas radiales para contribuir a la sensibilización de la comunidad universitaria acerca de estas problemáticas. Se adquirió hardware y software para permitir la digitalización y accesibilización de todo tipo de textos, los cuales son provistos gratuitamente en el soporte que la persona con discapacidad necesita. Se organizó la capacitación de los bibliotecarios y usuarios para posibilitarles el uso de estas herramientas. También se brindan audio grabaciones de clases o textos,

\footnotetext{
${ }^{4}$ Sitio Web: www.unlu.edu.ar
} 
de acuerdo con las necesidades. Se realizaron cursos de lengua de señas para el personal no docente, especialmente aquellos comprometidos en la atención a los estudiantes. Se participó del Programa "Podes" que, desde el Ministerio de Educación y Deportes de la Nación, otorgó notebooks con software y hardware adaptados a las necesidades de cada estudiante con discapacidad. En cuanto al eje de Accesibilidad pedagógica, se brindó asesoramiento individualizado a Coordinadores de Carreras, docentes, no docentes y estudiantes, respondiendo a las demandas para cada caso.

\section{METODOLOGÍA}

El objetivo de esta investigación fue indagar respecto del impacto de las acciones que se llevan a cabo en la UNLu para mejorar y fortalecer la integración de los estudiantes que se declaran en situación de discapacidad. Evaluamos el impacto en términos de la disminución del abandono, el rendimiento académico y la tasa de graduación.

Se trabajó con los ingresantes de las cohortes 2010 a 2015, para los cuales se disponía del relevamiento de quienes se declararon en situación de discapacidad al momento de su inscripción.

Se realizó un estudio cuantitativo, desde fuentes secundarias de información, utilizando la información del Sistema de Control Académico que utiliza la UNLu para administrar las carreras de grado y pregrado y el relevamiento efectuado por la Comisión de Accesibilidad respecto de los estudiantes que se declaran en situación de discapacidad.

Para estas 6 cohortes, se relevó la cantidad de ingresantes e identificamos a los estudiantes en situación de discapacidad. A los efectos de estudiar el abandono, se categorizó la situación de cada estudiante como activo, egresado o abandono. Para la categoría egresados, se tomó como criterio que si un estudiante obtuvo un título intermedio y no continúa cursando estaba incluido en esta categoría y no como abandono por haber obtenido una certificación académica.

Se evaluó el rendimiento académico, comparándolo con el total de la población de la universidad, de los estudiantes de estas 6 cohortes, considerando la Cantidad de Materias Aprobadas durante el período, el Promedio de Calificaciones y la Cantidad de Asignaturas Promovidas 
sobre el total cursadas. Este último indicador debido a que la UNLu tiene previsto en su Régimen General de Estudios la posibilidad de promoción sin examen final. Acceden a este sistema aquellos estudiantes que aprueban todas las evaluaciones previstas en una asignatura, sin desaprobar ninguna y con calificación promedio no inferior a 6 puntos y rinden una evaluación de carácter integrador con calificación no inferior a 7 puntos.

La población en estudio, comprendida por las cohortes 2010 a 2015, se integró con 30.072 ingresantes, de los cuales se identificó que 69 se declararon en situación de discapacidad, ya sea al momento de su inscripción o ante la Comisión Permanente, lo que representa el $0,23 \%$ del total de ingresantes.

\section{RESULTADOS ALCANZADOS}

Delimitada la población en estudio, se identificó el estado en que se encontraba cada estudiante al primer cuatrimestre de 2016. La Tabla 1 muestra la cantidad de estudiantes que se encuentran en cada categoría: activo o egresado y abandono, observándose que la tasa de abandono acumulado era del $64 \%$ de la matrícula. Al mismo tiempo, se observa que la tasa de abandono para quienes declararon situación de discapacidad es notoriamente inferior a la de los demás estudiantes con una diferencia a favor del $28 \%$ ( $36 \%$ vs $64 \%$ ).

Tabla 1. Situación al Año 2016 de los Ingresantes 2010-2015

\begin{tabular}{lccrrr}
\multirow{2}{*}{ SITUACIÓN } & \multicolumn{3}{c}{ CONDICION EN LA UNIVERSIDAD } & \multirow{2}{*}{ TOTAL } \\
\cline { 2 - 5 } & $\begin{array}{c}\text { ACTIVOS + } \\
\text { EGRESADOS }\end{array}$ & \multicolumn{1}{c}{$\%$} & ABANDONO & $\%$ & \\
\hline No declara Discapacidad & 10.784 & $35,94 \%$ & 19.219 & $64,06 \%$ & 30.003 \\
Declara Discapacidad & 39 & $56,52 \%$ & 30 & $43,48 \%$ & 69 \\
Total Población & $\mathbf{1 0 . 8 2 3}$ & $\mathbf{3 5 , 9 9 \%}$ & $\mathbf{1 9 . 2 4 9}$ & $\mathbf{6 4 , 0 1 \%}$ & $\mathbf{3 0 . 0 7 2}$ \\
Fuente: Elaboración Propia & & & & &
\end{tabular}

Dado que el tiempo trascurrido desde el momento del ingreso a la fecha, de corte del trabajo, difiere para cada cohorte, se diferenció el abandono en temprano y tardío, de manera tal de comparar el fenómeno en función del momento en que el estudiante deja la universidad. 
Recordemos que el abandono temprano es el que se produce durante los primeros dos años de estudio, mientas que el tardío es el que se produce con posterioridad a ese momento.

La Tabla 2, muestra que el abandono temprano de los estudiantes que no se declaran en situación de discapacidad al momento del ingreso

Tabla 2. Abandono Temprano y Tardío Cohortes 2010-2015

\begin{tabular}{lcccc}
\multirow{2}{*}{ POBLACIÓN } & \multicolumn{4}{c}{ POBLACIÓN } \\
\cline { 2 - 5 } & $\begin{array}{c}\text { No Declara } \\
\text { Discapacidad }\end{array}$ & $\%$ & $\begin{array}{c}\text { Declara } \\
\text { Discapacidad }\end{array}$ & $\%$ \\
\hline Abandono Temprano & 14.141 & $47,13 \%$ & 16 & $23,19 \%$ \\
Abandono Tardío & 5.078 & $16,92 \%$ & 14 & $20,29 \%$ \\
Abandono TOTAL & $\mathbf{1 9 . 2 1 9}$ & $\mathbf{6 4 , 0 6 \%}$ & $\mathbf{3 0}$ & $\mathbf{4 3 , 4 8 \%}$
\end{tabular}

Fuente: Elaboración Propia

Con los resultados obtenidos, a esta altura del análisis de la información relevada, se podría afirmar que el mayor impacto de las políticas implementadas como acciones de apoyo a los estudiantes que se declaran en situación de discapacidad, las que son coordinadas por la Comisión Permanente de Seguimiento de la Accesibilidad, se da durante los primeros años de estudio dado que el abandono temprano de estos ingresantes es aproximadamente la mitad que el que se da en aquellos que no se declararon en situación de discapacidad. es del $47,13 \%$, mientras que el de quienes se declaran en situación de discapacidad es del $23,19 \%$. Respecto del abandono tardío, el comportamiento es inverso dado que en quienes se declararon en situación de discapacidad llega al $20 \%$ y en quienes no al $16,92 \%$. 
mejor desempeño por parte de los ingresantes que se declararon en situación de discapacidad. Estos estudiantes muestran, en promedio, una diferencia significativa respecto de la cantidad de materias aprobadas.

También una mayor tasa de promoción, que es el cociente entre las asignaturas aprobadas mediante el régimen de promoción sin examen final y la totalidad de asignaturas cursadas.

Respecto del promedio de calificaciones, la diferencia no es tan significativa pero sigue siendo mayor

Tabla 3. Indicadores de Rendimiento Académico Cohortes 2010-2015

\begin{tabular}{lcc}
\multicolumn{1}{c}{ INDICADOR } & Sin Discapacidad & Con Discapacidad \\
\hline Materias Aprobadas & 4,47 & 6,35 \\
Tasa de Promoción & 2,66 & 3,30 \\
Promedio Calificaciones & 4,03 & 4,44
\end{tabular}

Finalmente, se calculó la tasa de egreso, observándose un mejor desempeño de quienes se declararon en situación de discapacidad dado que la tasa de egreso para las cohortes 2010-2015, calculada al primer cuatrimestre de 2016 , es del $4,55 \%$ mientras que para el resto de los integrantes de estas cohortes es del $1,84 \%$.

Para observar, con mayor claridad, el desempeño de ambos grupos de estudiantes se graficaron los indica- para el grupo de estudiantes que contaron con el apoyo y seguimiento de la Comisión Permanente y el Servicio Social de la UNLu.

También se detectó que las carreras con mayor proporción de estudiantes que declaran situación de discapacidad, para el período que abarcó el estudio, son la Licenciatura en Trabajo Social, Licenciatura en Sistemas de Información, Profesorado en Historia y Tecnicatura Universitaria en Industrias Lácteas.
Promedio Calificaciones

Fuente: Elaboración Propia dores calculados, agregando la cantidad de años de permanencia en la institución para representar la tasa de abandono. El Gráfico 1 muestra la relación de cada indicador para los estudiantes que se declararon en situación de discapacidad respecto de aquellos que no se declararon en esa situación. 


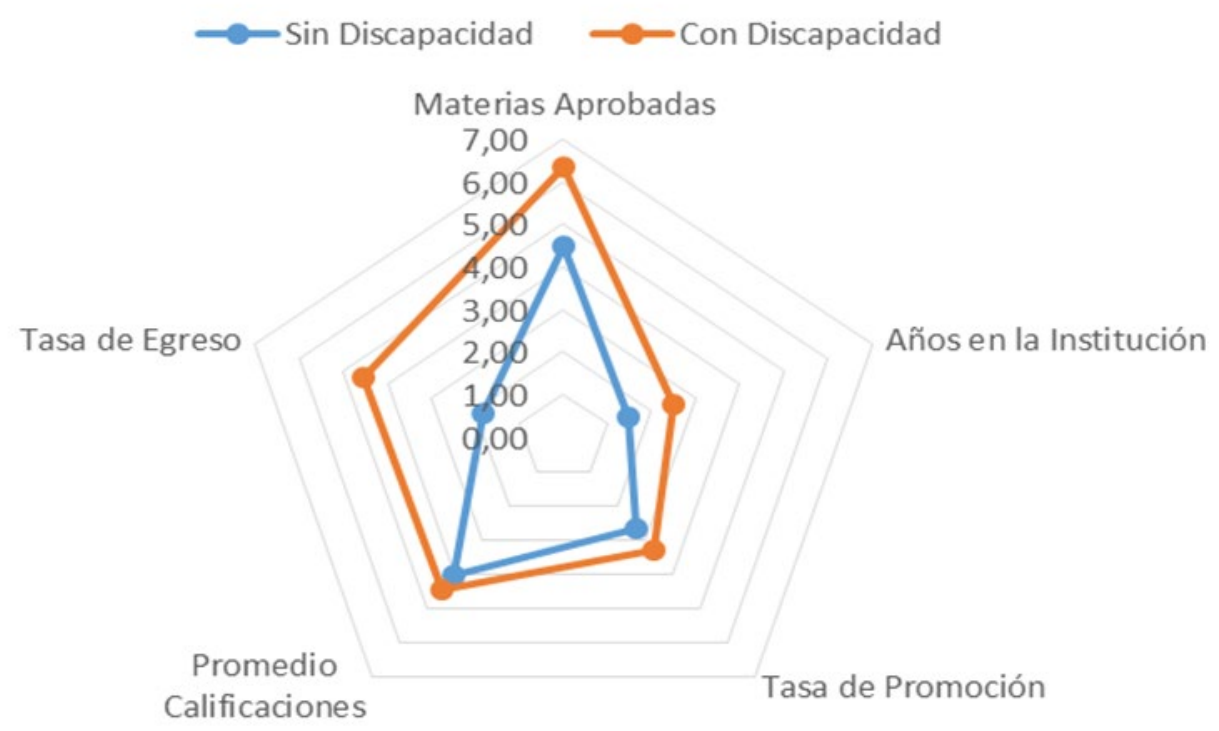

Figura 1: Indicadores de Rendimiento, Egreso y Abandono Cohortes 2010-2015

CONCLUSIONES

En términos del objetivo planteado para este trabajo, se verificó que las acciones que lleva a cabo la Comisión Permanente de Seguimiento de la Accesibilidad, sumadas al Programa de Becas Estudiantiles de la UNLu, resultan efectivas para la retención y la mejora del rendimiento académico de aquellos estudiantes que se declaran en situación de discapacidad al comenzar los estudios universitarios.

Resulta alentador ver cómo estas acciones que tienden a la contención y apoyo de los sectores más desfavorecidos que pueden acceder a la educación superior gracias a la gratuidad de la enseñanza y el ingreso irrestricto, en la República Argentina, sirven para consolidar esta posibilidad de movilidad social y que la misma no sea un mero eslogan o la falsa ilusión de que es posible acceder pero imposible permanecer o graduarse.

Como recomendación final, creemos que es importante fortalecer y extender estas acciones de apoyo a diversos grupos de estudiantes de manera tal de propender a la disminución de los altos índices de abandono temprano que se observan en el Sistema de Educación Superior. De todas maneras, se cree conveniente que se trabaje en clasificar claramente los motivos por los cuales los estudiantes dejan los estudios superiores para no contabilizar como abandono aquellos casos en que por propia decisión de vida el estudiante decide dejar la universidad, ya sea para cursar estudios de otro tipo o para dedicarse a la actividad laboral dado 
que no requiere una certificación académica para el ejercicio de la misma.

\section{REFERENCIAS}

Convención sobre los Derechos de las

Personas con Discapacidad (2008). Ley Honorable Congreso de la Nación Argentina № 26.378. Publicada en el Boletín Oficial del 09-jun-2008 Número: 31422 Página: 1

Decreto 84 (2014), "Programa de Respaldo a Jóvenes Argentinos". Presidencia de la Nación, República Argentina. Publicado en el Boletín Oficial de la Nación $\mathrm{N}^{\circ} 32.814$ del 27 de Enero de 2014.

Ley de Educación Superior (1995). Ley 24.521/1995 del Honorable Congreso de la Nación Argentina. Promulgada parcialmente el 7 de Agosto de 1995.

Ley de Educación Nacional (2006). Ley $\mathrm{N}^{\circ}$ 26.206/2006 del Honorable Congreso de la Nación Argentina. Promulgada el 27 de Diciembre de 2006.

Librandi, Ariel y Katz, Sandra. (2014). Inclusión en la Educación Superior. Revista Latinoamericana de Educación Inclusiva. Revista
Latinoamericana de Educación Inclusiva, Vol. 8, $\mathrm{N}^{\circ} 1$, de marzo 2014.

Oloriz, M.; Fernandez, J.M.; Amado, V. (2013) Impacto del Programa de Becas de la Universidad Nacional de Luján en la Disminución del Abandono. III Conferencia Latinoamericana sobre el Abandono en la Educación Superior. CLABES 2013, UNAM, México.

Oloriz, M.; Fernandez, J.M.; Amado, V. (2014) Comparación entre el Programa de Becas Estudiantiles de la Universidad Nacional de Luján y los Programas de Becas Nacionales. IV Conferencia Latinoamericana sobre el Abandono en la Educación Superior. CLABES 2014, Universidad de Antioquía, Colombia.

Oloriz, M.; Fernandez, J.M.; Rodríguez, R. (2015). Impacto del "Programa de Respaldo a Estudiantes Argentinos" en la Disminución del Abandono en la Universidad Nacional de Luján. $\checkmark$ Conferencia Latinoamericana sobre el Abandono en la Educación Superior. CLABES 
2015, Universidad de Talca,

Chile

ONU. (2006) "Convención de los

Derechos de las Personas con

Discapacidad”, Diciembre 2006.

Peralta, A. (2007). Libro Blanco sobre universidad y discapacidad. Madrid: Real Patronato sobre Discapacidad

UNESCO (2003). Superar la exclusión mediante planteamientos integrales en la educación. Un desafío \& una visión. Documento Conceptual. En línea en http://unesdoc.unesco.org/imag es/0013/001347/134785s.pdf Acceso: Julio 2016.

UNLu, (2005). Programa de Becas Estudiantiles de la Universidad Nacional de Luján aprobado por Res. HCS No 103/05.

UNLu, (2008). Creación de la Comisión Permanente de Seguimiento de la Accesibilidad de la Universidad Nacional de Luján. Aprobado por Res. HCS $N^{\circ}$ 660/08. 